\title{
Crystal structure of tri(barium, strontium) tetragermanide, $\mathrm{Ba}_{2.62} \mathrm{Sr}_{0.38} \mathrm{Ge}_{4}$
}

\author{
F. Zürcher and R. Nesper* \\ ETH Zürich, Laboratorium für Anorganische Chemie, Universitätstraße 6, CH-8092 Zürich, Switzerland
}

Received February 23, 1999, transferred to 2nd update of database ICSD in 1999, CSD-No. 409406

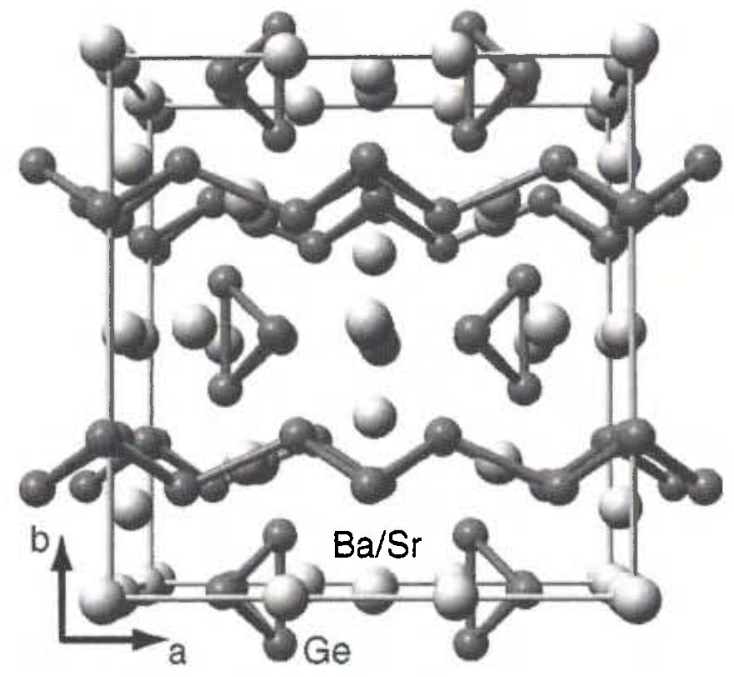

Abstract

$\mathrm{Ba}_{2.62} \mathrm{Ge}_{4} \mathrm{Sr}_{0.38}$, orthorhombic, Cmmm (No. 65), $a=11.773(8) \AA$, $b=12.126(8) \AA, c=11.950(8) \AA, V=1706.0 \AA^{3}, Z=8$, $R_{\mathrm{gt}}(F)=0.027, w R\left(F^{2}\right)=0.070, T=298 \mathrm{~K}$.

\section{Source of material}

$\mathrm{Ba}_{2.62} \mathrm{Sr}_{0.38} \mathrm{Ge}_{4}$ is prepared by heating a mixture of the elements with a $\mathrm{Ba} / \mathrm{Sr} / \mathrm{Ge}$ ratio of 2.5:0.5:4 at $1273 \mathrm{~K}$ (12 h, cooling down within $5 \mathrm{~h}$ ). $\mathrm{Ba}_{2.62} \mathrm{Sr}_{0.38} \mathrm{Ge}_{4}$ forms grey-black, plate-like crystals with a metallic lustre.

The $\mathrm{Ba} / \mathrm{Sr} 3, \mathrm{Ba} / \mathrm{Sr} 4$, and $\mathrm{Ba} / \mathrm{Sr} 5$ sites are occupied by different amounts of barium and strontium. The corresponding occupancy factors are also refined.

\section{Discussion}

$\mathrm{Ba}_{2.62} \mathrm{Sr}_{0.38} \mathrm{Ge}_{4}$ crystallises with the $\mathrm{Ba}_{3} \mathrm{Ge}_{4}$ structure type [1]. It contains isolated $\mathrm{Ge}_{4}{ }^{6-}$ and polymerized ${ }_{\infty}^{1}\left[\mathrm{Ge}_{4}{ }^{6-}\right]$ butterfly anions. $\mathrm{Ba}_{2.62} \mathrm{Sr}_{0.38} \mathrm{Ge}_{4}$ can be described, according to the Zintl-Klemm concept, by the formulation $\left(\mathrm{M}^{2+}\right)_{6}\left[\mathrm{Ge}_{4}{ }^{6-}\right]_{-x}^{1}\left[\mathrm{Ge}_{4}{ }^{6-}\right]$ $\left(\mathrm{M}=\mathrm{Ba}, \mathrm{Sr}\right.$ ). The $\mathrm{Ba}_{3} \mathrm{Ge}_{4}$ structure can be also described as (distorted) cubic close packing of Zintl anions with all tetrahedral and octrahedral holes occupied by metal atoms. In $\mathrm{Ba}_{2.62} \mathrm{Sr}_{(0.38} \mathrm{Ge}_{4}$, the larger octahedral positions $\mathrm{M} 1$ and $\mathrm{M} 2$ are exclusively occupied by barium, while the strontium amount on the smaller tetrahedral positions M3 to M5 is $18 \%, 21 \%$, and $18 \%$, respectively. The main characteristic of $\mathrm{Ba}_{3} \mathrm{Ge}_{4}$ is the presence of an unusually long intermolecular bond ( $2.87 \AA$ ). In $\mathrm{Ba}_{2.62} \mathrm{Sr}_{0.38} \mathrm{Ge}_{4}$, this bond is even more longer ( $2.91 \AA$ ) although, as expected, the cell volume is smaller than in $\mathrm{Ba}_{3} \mathrm{Ge}_{4}$.

Table 1. Data collection and handling.

\section{Crystal:}

Wavelength:

$\mu$ :

Diffractometer, scan mode:

$2 \theta_{\text {max }}$ :

$N(h k l)_{\text {measured, }} N(h k l)_{\text {unique: }}$ Criterion for $I_{\mathrm{obs}}, N(h k l)_{\mathrm{gt}}$

$N(\text { param })_{\text {refined: }}$

Programs:

Table 2. Atomic coordinates and displacement parameters (in $\AA^{2}$ ).

\begin{tabular}{|c|c|c|c|c|c|c|c|c|c|c|c|}
\hline Atom & Site & Occ. & $x$ & $y$ & $z$ & $U_{11}$ & $U_{22}$ & $U_{33}$ & $U_{12}$ & $U_{13}$ & $U_{23}$ \\
\hline $\mathrm{Ba}(1)$ & 4 & & 0 & $0.16057(8)$ & $1 / 2$ & $0.0194(5)$ & $0.0223(5)$ & $0.0270(6)$ & 0 & 0 & 0 \\
\hline $\mathrm{Ba}(2)$ & $8 g$ & & $0.66281(8)$ & 0 & 0 & $0.0166(5)$ & $0.0208(5)$ & $0.0274(6)$ & 0 & 0 & 0 \\
\hline $\mathrm{Ba}(3)$ & $4 k$ & $0.82(2)$ & 0 & 0 & $0.16059(8)$ & $0.0186(6)$ & $0.0213(6)$ & $0.0173(6)$ & 0 & 0 & 0 \\
\hline $\operatorname{Sr}(3)$ & $4 k$ & 0.18 & 0 & 0 & 0.16059 & 0.0186 & 0.0213 & 0.0173 & 0 & 0 & 0 \\
\hline $\mathrm{Ba}(4)$ & $4 l$ & $0.79(2)$ & 0 & $1 / 2$ & $0.32525(8)$ & $0.0162(6)$ & $0.0227(6)$ & $0.0158(7)$ & 0 & 0 & 0 \\
\hline $\operatorname{Sr}(4)$ & $4 l$ & 0.21 & 0 & $1 / 2$ & 0.3252 & 0.0162 & 0.0227 & 0.0158 & 0 & 0 & 0 \\
\hline $\mathrm{Ba}(5)$ & $8 m$ & $0.82(1)$ & $1 / 4$ & $1 / 4$ & $0.25556(6)$ & $0.0189(5)$ & $0.0218(5)$ & $0.0180(5)$ & $0.0003(3)$ & 0 & 0 \\
\hline $\operatorname{Sr}(5)$ & $8 m$ & 0.18 & $1 / 4$ & $1 / 4$ & 0.25556 & 0.0189 & 0.0218 & 0.0180 & 0.0003 & 0 & 0 \\
\hline $\operatorname{Ge}(1)$ & $8 q$ & & $0.1938(1)$ & $0.39389(9)$ & $1 / 2$ & $0.0201(7)$ & $0.0208(7)$ & $0.0194(6)$ & $-0.0019(4)$ & 0 & 0 \\
\hline $\operatorname{Ge}(2)$ & $8 p$ & & $0.1370(1)$ & $0.20163(9)$ & 0 & $0.0215(7)$ & $0.0203(6)$ & $0.0184(6)$ & $0.0008(5)$ & 0 & 0 \\
\hline $\operatorname{Ge}(3)$ & $8 n$ & & 0 & $0.29310(9)$ & $0.1427(1)$ & $0.0167(6)$ & $0.0240(6)$ & $0.0245(7)$ & 0 & 0 & $-0.0042(5)$ \\
\hline $\mathrm{Ge}(4)$ & 80 & & $0.1986(1)$ & 0 & $0.3471(1)$ & $0.0213(7)$ & $0.0228(6)$ & $0.0236(7)$ & 0 & $-0.0059(5)$ & 0 \\
\hline
\end{tabular}

\footnotetext{
* Correspondence author (e-mail: nesper@inorg.chem.ethz.ch)
} 
Acknowledgment. This work was supported by the Swiss National Foundation under project no. 2000-050675.97

\section{References}

1. Zürcher, F.; Nesper, R.: Ba ${ }_{3} \mathrm{Ge}_{4}$ : Polymerization of Zintl Anions in the Solid and Bond Stretching Isomerism. Angew. Chem. Int. Ed. 37 (1998) 3314-3318.
2. Sheldrick, G. M.: SHELXS-96. Program for the solution of crystal structures. University of Göttingen, Germany 1996.

3. Sheldrick, G. M.: SHELXL-96. Program for refining crystal structures. University of Göttingen, Germany 1996.

4. Hofmann, P.; Nesper, R.: COLTURE 3-D Color Graphic Program. ETH Zürich, Switzerland 1995. 